A

Aalto University

School of Science

\title{
Algorithmic surface extraction from MRI data: modelling the human vocal tract
}

\author{
D. Aalto, J. Helle, A. Huhtala, A. Kivelä, J. Malinen, J. Saunavaara, and T. Ronkka \\ Aalto University, University of Helsinki, Medical Imaging Centre of Southwest Finland
}

\section{Introduction}

A novel method is proposed for efficient extraction of high quality vocal tract (VT) surface models from MRI data. Our motivation stems from computational acoustics modelling of the VT $[1,2]$. 3D models can be used for other applications, such as physical reconstructions of the VT for medical studies and oral and maxillofacial surgery planning.

Problems arise from osseous structures being indistinguishable from air in MRI data. This causes the teeth and maxillae to merge into the VT air column in image data as seen in Fig. 3a. Resulting artefacts are masked using a separate model that is aligned with the VT geometry.

\section{Surface extraction and artefact modelling}
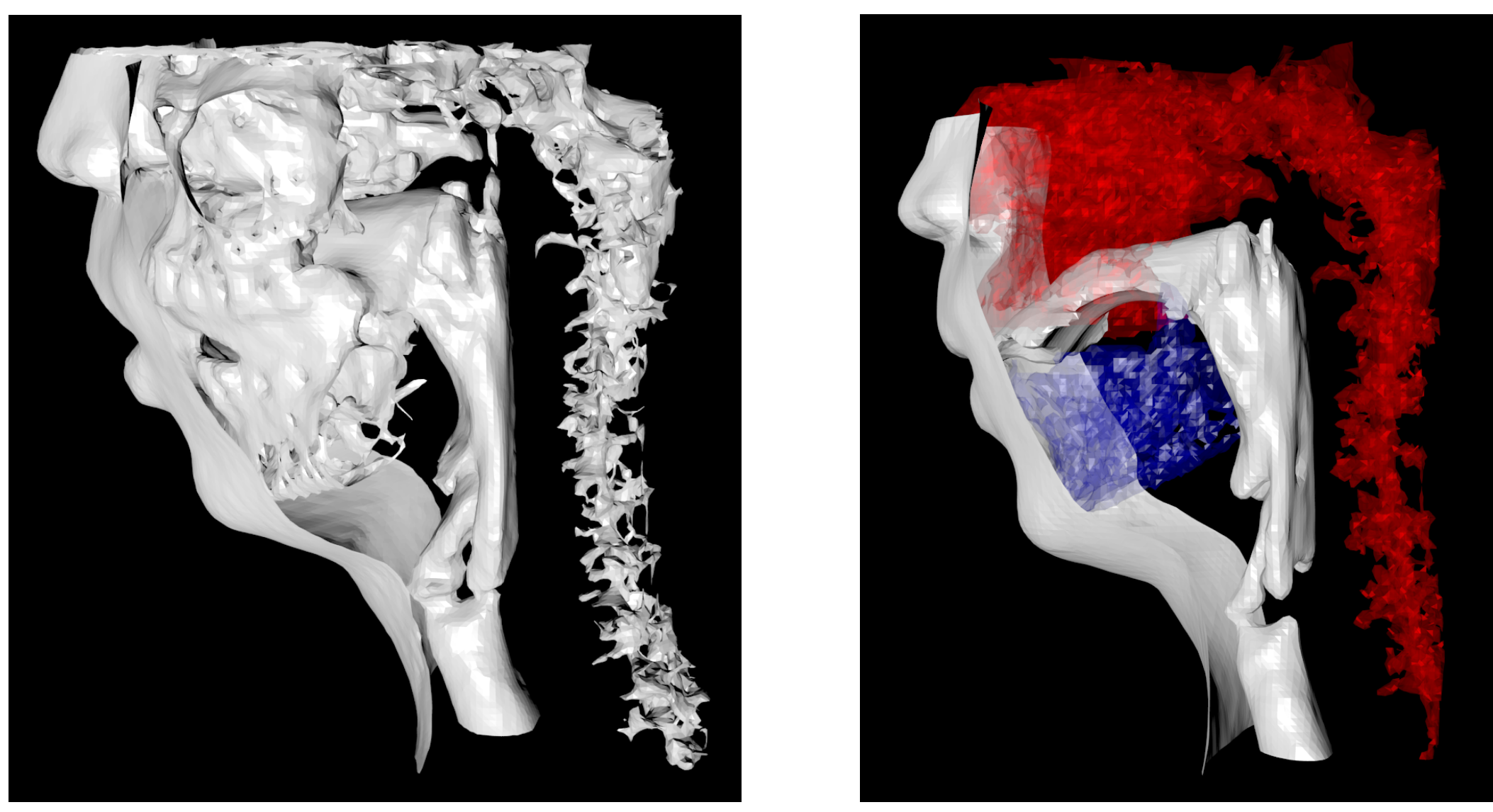

Figure 1: Extracted surface before and after artefact removal. (a) Initial surface; (b) Artefacts identified.

The outline of the algorithm is as follows:

1. Extract a preliminary air-tissue interface from the data (Fig. 1a).

2. Identify the artefacts from the surface mesh (Fig. 1b).

- The same artefact model can be used for all VT configurations from the same subject.

3. Align the artefact model using Point Cloud Library [3].

4. Mask out the unwanted volume using the alignment information.

5. Re-extract the final air-tissue interface from the masked data.

The VT has a fairly complicated and variable shape. Even the nasal tract should be part of the extracted volume for some speech geometries. For better accuracy, artefact models could be produced by imaging the teeth separately and by creating MRI visible markers for the alignment of the two models.
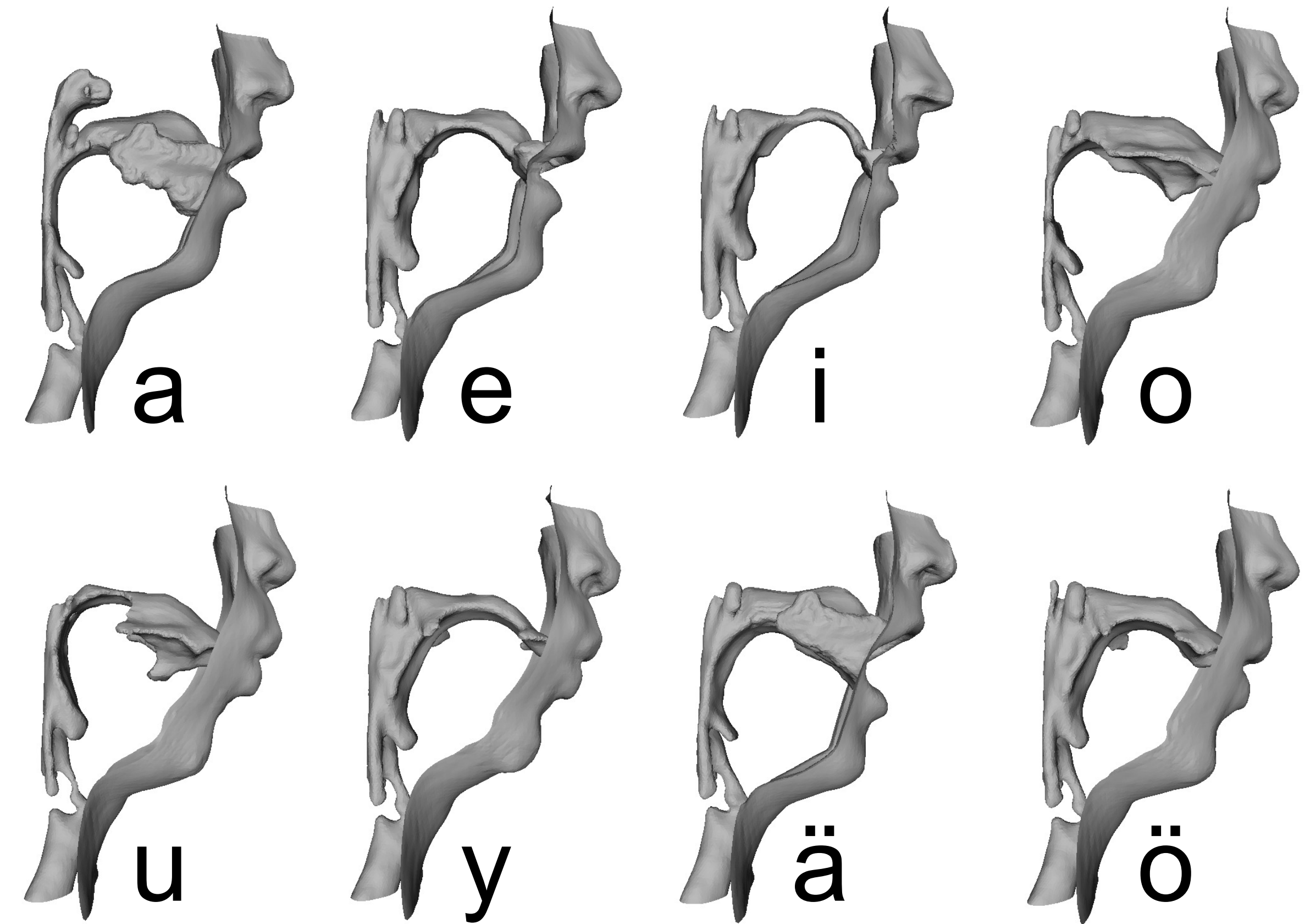

Figure 2: Final surface meshes for all Finnish vowels.
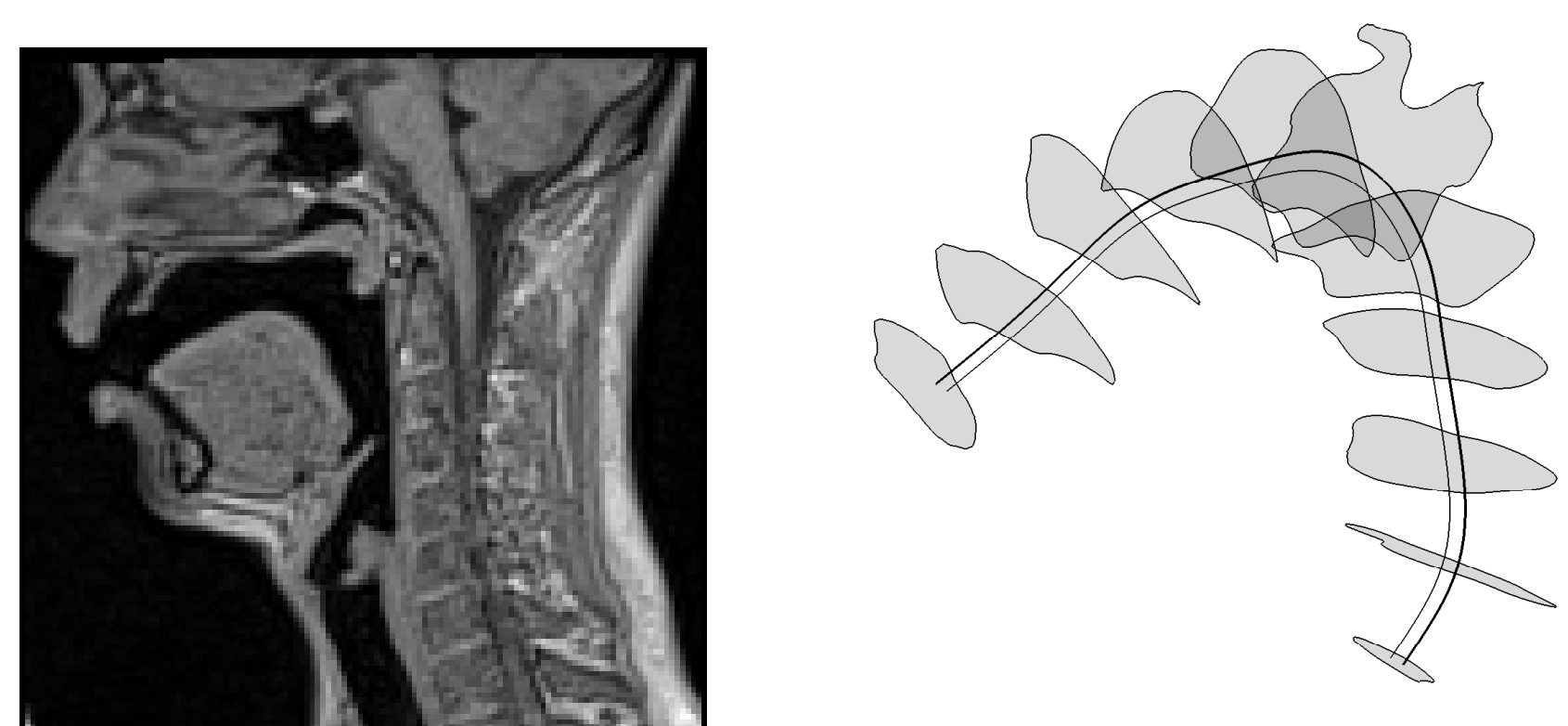

Figure 3: (a) Mid-sagittal plane of MRI; (b) Intersections of the VT perpendicular to a centreline.

\section{Centrelines}

Vocal tract centrelines are required for some computational speech models. Unfortunately, the "acoustic" centreline is not well-defined on geometric grounds alone. Hence, practical modelling requires parameter tuning based on choosing from families of centrelines, see Fig. 3b. To construct the nominal centreline, we solve a steady state heat equation inside the VT volume. Similar data processing is carried out, e.g., in modelling vascular structures [4].

\section{Physical models}

We have used the 3D models for experiments with rapid prototyping. The printout shown in Fig. 4 has been produced using an inexpensive fused deposition $3 \mathrm{D}$ printer. The VT is a non-convex shape, and, as such, it is challenging to print using additive printing technologies.

Other 3D printing technologies, such as stereolithography, can be used to produce higher quality printouts without significant effort in data processing. However, the unit price may then be prohibitively high since multiple models of the same subject are required.

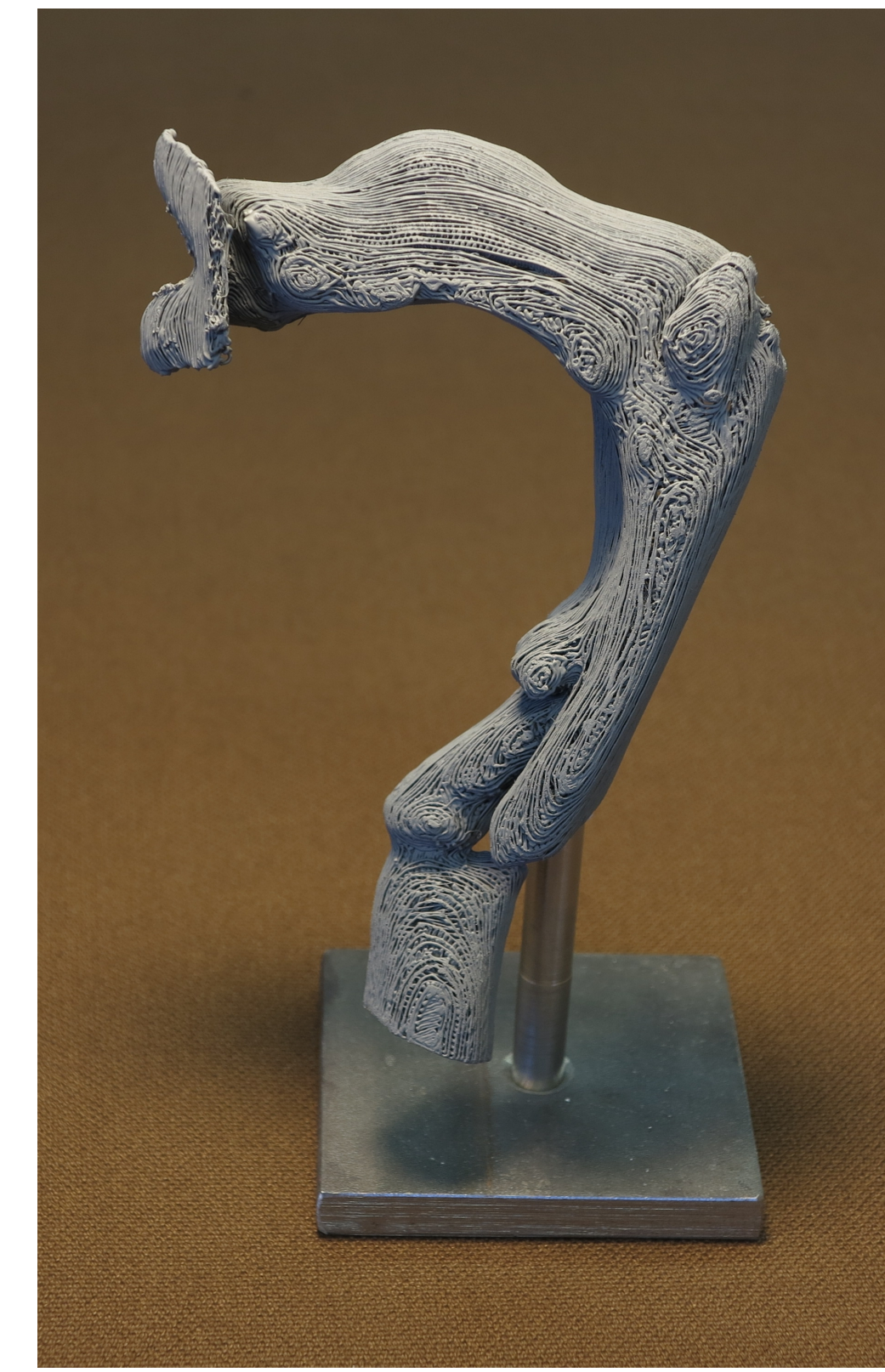

Figure 4: $3 D$ printed VT

\section{Conclusion}

The proposed method can be used to extract vocal tract geometries with reasonable manual work. More robust artefact removal can be done by separately imaging the teeth and producing markers to help in the alignment of the models.

3D models for the VT have many applications. Rapid prototyping techniques are nowadays affordable so that inexpensive reconstructions can be produced for clinical use. When combined with computational models, physical reconstruction are expected to have applications, e.g., in surgery planning.

\section{References}

[1] Aalto, D., Aaltonen, O., Happonen, R.-P., Malinen, J., Palo, P., Parkkola, R., Saunavaara, J., and Vainio, M. (2011). Recording speech sound and articulation in MRI. In Proc. BIODEVICES 2011, Rome.

[2] Hannukainen, A., Lukkari, T., Malinen, J., and Palo, P. (2007). Vowel formants from the wave equation. J. Acoust. Soc. Am. Express Letters, 122(1):EL1-EL7.

[3] Rusu, R. B. and Cousins, S. (2011). 3D is here: Point Cloud Library (PCL). In IEEE International Conference on Robotics and Automation (ICRA), Shanghai.

[4] Antiga, L. (2003). Patient-Specific Modeling of Geometry and Blood Flow in Large Arteries. PhD thesis, Politecnico di Milano. 